(c) American Dairy Science Association, 2005.

\title{
Escherichia coli Strain Nissle 1917: Significant Reduction of Neonatal Calf Diarrhea
}

\author{
R. von Buenau, ${ }^{1}$ L. Jaekel, ${ }^{2}$ E. Schubotz, ${ }^{3}$ S. Schwarz,${ }^{4}$ T. Stroff, ${ }^{5}$ and M. Krueger ${ }^{6}$ \\ ${ }^{1}$ Dr. Ponsold GmbH, Department of Animal Health, Breitscheidstrasse 28a, \\ 39387 Oschersleben, Germany \\ 2Jonastal 8, 99310 Arnstadt, Germany \\ ${ }^{3} \mathrm{~F}$ 41, 04523 Elstertrebnitz, Germany \\ ${ }^{4} \mathrm{Am}$ Silberberg 10, 08396 Oberwiera, Germany \\ ${ }^{5}$ Holzwickeder Str. 49a, 59427 Unna, Germany \\ ${ }^{6}$ Institute for Bacteriology and Mycology, Veterinary Medicine, \\ University of Leipzig, An den Tierkliniken 29, 04103 Leipzig, Germany
}

\section{ABSTRACT}

Inappropriate daily use of antimicrobial drugs for the treatment of intestinal diseases is associated with an increased risk of antibiotic resistance. Thus, the establishment of new forms of therapy is still needed. Our objective was to examine the effect of the nonpathogenic Escherichia coli strain Nissle 1917 on the prophylaxis and treatment of neonatal calf diarrhea in a hypothesisgenerating study (study I) and a subsequent confirmatory clinical study (study II) under field conditions. Both trials were designed as consecutive, placebo-controlled, single-blind comparisons of 2 groups of animals. Immediately after birth, healthy calves were assigned to either the $E$. coli Nissle 1917 or the placebo group. The study medication was administered orally $1 / \mathrm{d}$ before the first feeding. The treatment was continued for the first 10 to $12 \mathrm{~d}$ of life. For each animal, the studies ended on d 20 to 22 of life. In both trials, the number of calves developing diarrhea was defined as the primary target criterion. A total of 335 newborn calves were included in the studies (study I: $\mathrm{n}=172$; study II: $\mathrm{n}=$ 163). Study I showed that the incidence of diarrhea was $65.2 \%$ under placebo and $26.5 \%$ under E. coli Nissle 1917. In study II, the corresponding figures were $63.0 \%$ under placebo and $12.2 \%$ under $E$. coli Nissle 1917 . It can be concluded that the administration of viable $E$. coli bacteria, strain Nissle 1917, has a clear beneficial effect on the prophylaxis and treatment of neonatal calf diarrhea.

(Key words: Escherichia coli strain Nissle 1917, neonatal calf diarrhea, Ponsocol)

Received September 12, 2003.

Accepted August 2, 2004.

Corresponding author: R. von Buenau; e-mail: vbuenau@ ardeypharm.de.

\section{INTRODUCTION}

Neonatal diarrhea is one of the main causes of calf death worldwide and also of financial loss in the cattle industry. The mean incidence of diarrhea in individual herds of cattle can be as high as $80 \%$ (Cornaglia et al., 1992; Wright et al., 1995); therefore, rates of diarrhea $>50 \%$ are not unusual. The mortality rate was reported to vary between 1.5 and $8 \%$ (Bendali et al., 1999), although rates up to $25 \%$ have been described (Frank and Kaneene, 1993). More than 52\% of diarrhea cases and about $60 \%$ of all losses occur within the first 7 to $10 \mathrm{~d}$ of life (Virtala et al., 1996; Bendali et al., 1999). The main cause of neonatal calf diarrhea is infectious disease of the intestine. Treatment of calf diarrhea is commonly based on the use of broad-spectrum antibiotics or other antimicrobials, either with or without concomitant liquid electrolyte therapy.

Before Sir Alexander Fleming's discovery of penicillin, disorders of the intestinal tract were frequently treated with viable nonpathogenic bacteria to change or replace the intestinal microflora. Today, probiotic treatment is increasingly becoming the focus of clinical interest (Abe et al., 1995; Avila et al., 1995; Kyriakis et al., 1999). Oral administration of bacteria may reduce the risk of intestinal diseases in humans (Salminen et al., 1996; Fric, 2002) and animals (Gedek, 1986; Reuter, 1997; Reid and Friendship, 2002). In Germany, Escherichia coli strain Nissle 1917 is authorized under the brand name Ponsocol for the prophylaxis of neonatal calf diarrhea and has been marketed since 2001.

This nonpathogenic strain has been well established in human medicine since 1917 (Nissle, 1918). Under the brand name Mutaflor, it is used successfully for treating various gut-related diseases, e.g., chronic constipation (Moellenbrink and Bruckschen, 1994), ulcerative colitis (Kruis et al., 1997; Rembacken et al., 1999), Crohn's disease (Malchow, 1997), or pouchitis (Kuzela et al., 2001). In addition, the strain prevents colonization of the intestine with microbial pathogens in new- 
born infants (Lodinová-Zádniková and Sonnenborn, 1997). The application of $E$. coli strain Nissle 1917 is well tolerated. Disadvantages and negative consequences, especially of long-term usage, are not known.

The strain is well characterized by means of microbiological, biochemical, and serological typing, as well as molecular genetics (Blum et al., 1995; StentebjergOlesen et al., 1999; Grozdanov et al., 2002). It is sensitive to all antibiotics directed against gram negative bacteria and can be differentiated from other bacteria via serological, microbiological, and molecular biological methods (Blum-Oehler et al., 2003). After introduction into newborns, the strain has colonized the intestine and remained genetically stable. In addition, in conjugation experiments it has been shown to be a poor recipient for foreign plasmid DNA.

In vitro the $E$. coli strain Nissle 1917 is antagonistically active against Salmonella $\mathrm{spp}$., Shigella ssp., some enteropathogenic E. coli, Proteus ssp., and Candida ssp. (Sonnenborn and Greinwald, 1991). This nonpathogenic $E$. coli strain displaces enteropathogenic $E$. coli, Salmonella typhimurium and Candida albicans in vivo (Lorenz and Schulze, 1996; Kuzela et al., 2001). In addition, the strain enhances the immune response against bacterial and fungal infections in vivo (Hockertz, 1997).

The objective of the 2 placebo-controlled studies presented here was to investigate the effect of oral administration of a suspension of viable $E$. coli strain Nissle 1917 on the prophylaxis and treatment of neonatal calf diarrhea under field conditions.

\section{MATERIALS AND METHODS}

\section{Sites and Animals}

The hypothesis-generating and subsequent confirmatory clinical trials were conducted from February to April 1998 and June to November 1998, respectively. To obtain adequate numbers of animals within a short period of time, 4 sites were selected, each with rearing facilities for 900 to 1400 calves/yr. The sites of both studies were located near Leipzig, Germany. All calves were "Deutsches Schwarzbuntes Rind" [German Black and White cattle] with 65 to $80 \%$ "Holstein-Friesian" genes.

\section{Animal Handling, Housing, and Treatment}

Animal handling and treatment were conducted in accordance with the German Animal Protection Act (Tierschutzgesetz) and approved by the local authorities. All calves included in the studies were kept separated from the rest of the animals. The management and feeding practices in all sites were nearly identical. All calves were born on the study sites. The dams were separated from the rest of the stock $12 \mathrm{~h}$ before calving. Each calf was fed immediately after birth a total of 2.5 to $3 \mathrm{~L}$ of the colostrum from its own dam with a nipple bottle. On d 2 and 3 , the calves were fed the same amount of $d 2$ and 3 colostrum. From $d 4$ to the end of the study, pooled milk or commercially available milk replacer (Kaelberstolz 15-Kristall, Salvana Tiernahrung GmbH, Elmshorn, Germany) was fed to the animals.

All animals were fed 5\% of their individual BW 3 times daily using individual buckets. Before feeding, $d$ 2 and 3 colostrum was preheated at 35 to $40^{\circ} \mathrm{C}$. Pooled milk was first preheated at $65^{\circ} \mathrm{C}$ for $30 \mathrm{~min}$ and then cooled to $35^{\circ} \mathrm{C}$. Milk replacer was used in accordance with the manufacturer's recommendations. After dissolving in preheated water at 42 to $45^{\circ} \mathrm{C}$, it was administered after cooling to $35^{\circ} \mathrm{C}$. The milk replacer contained $20 \%$ protein, $15 \%$ fat, $1.6 \%$ lysine, $1 \% \mathrm{Ca}, 0.8 \%$ $\mathrm{P}, 0.5 \%$ fiber, and other ingredients (vitamins $\mathrm{A}, \mathrm{D}$, and $\mathrm{E}, \mathrm{Cu}$, and others) in lower quantities. Dry food (straw and hay) and water were accessible ad libitum.

All newborn calves, except those born late at night, were separated immediately from their dams. Calves born late at night were separated by the next morning at the latest. All calves were housed for $3 \mathrm{~d}$ in individual pens. From d 4 until the end of the trial, all animals were kept in groups containing a maximum of 10 individuals. Single pens and group pens covered an area of 1.5 and $60 \mathrm{~m}^{2}$, respectively. Contact of the calves between the pens was not possible. Calves in single pens were housed on concrete or tiles covered with straw or rubber mats, whereas in the group pens, only straw was used as a covering material. Pens were cleaned and disinfected after every usage (in-out system). Calves not suitable for the studies were housed and, in case of illness, nursed separately.

The study medication was given orally, once per day, on $\mathrm{d} 1$ before the first suckling and from $\mathrm{d} 2$ to 10 before the first feeding in the morning. Each calf received 15 $\mathrm{mL}$ of $E$. coli strain Nissle 1917 or placebo suspension per day. Escherichia coli strain Nissle 1917 suspension ingredients included $1 \times 10^{8} / \mathrm{mL}$ of viable $E$. coli bacteria, strain Nissle 1917, $\mathrm{NaCl}, \mathrm{KCl}, \mathrm{MgSO}_{4}, \mathrm{CaCl}_{2}$, $\mathrm{MgCl}_{2}$, and water. The placebo suspension contained only the listed salts, dissolved in water.

\section{Experimental Design}

Both studies were conducted as a consecutive, placebo-controlled, single-blind comparison of 2 groups. The consecutive design was chosen to avoid transmission of bacteria of $E$. coli strain Nissle 1917 from animals of the $E$. coli strain Nissle 1917 group to the placebo group. At first, calves were assigned to the placebo 
group. After reaching the required number of animals, inclusion was stopped until the last calf of the group had completed the trial. In a second step, the animals of the E. coli strain Nissle 1917 group were included, treated, and observed. To keep both treatment groups comparable, all animals were recruited in the same facilities and the same herds of cattle. Management was kept unchanged. Disease outbreak common to one group did not occur.

To overcome the time difference required to avoid cross-contamination, the length of the study at each site was kept as short as possible by choosing facilities with a rate of 2 to 3 calvings/d. Except at one site in study II, at the most, 6 to $8 \mathrm{wk}$ from recruiting the first calf to the end of the study of the last calf were needed. Only animals that were born healthy, without requiring considerable birth assistance, and with a BW of at least $33 \mathrm{~kg}$ were included in the studies.

All calves were treated with either $E$. coli strain Nissle 1917 or placebo for the first $12 \mathrm{~d}$ of life in the hypothesis-generating trial and for the first $10 \mathrm{~d}$ of life in the confirmatory trial. All animals included in the trials were then examined twice per day for an additional 10 d. Control examinations were conducted twice daily to record the consistency of feces, tolerance to treatment, occurrence of adverse events, newly occurring exclusion criteria, newly developed illnesses, state of general health, and the use of additional medication.

In the 2 studies, the primary target criterion was the number of calves developing diarrhea. For assessing the difference of severity of diarrhea, the consistency of calf feces was classified as follows: 1) normal; 2) soft but retaining its form; 3 ) loose, not retaining its form, or 4 ) watery. Calves with feces classified as 3 or 4 on $\geq 2$ consecutive days were regarded as cases of diarrhea. Shorter durations were not assessed as cases of diarrhea. To avoid effects induced by concomitant medication, antidiarrhetic treatment, except for antimicrobial rescue medication, was conducted in the following manner: On $d 1$ (the day of suspected diarrhea) colostrum, milk, or milk replacer was discontinued, and only electrolytes or dietetic treatment, or both, were given. On d 2 (the day of diagnosis) symptomatic therapies with electrolytes, adstringent, or dietetic treatment were administered. On d 3, an additional application of antimicrobial drugs was given, if necessary.

As secondary efficacy criteria, the occurrence of diseases other than diarrhea, use of concomitant medication, and mortality were documented.

If diarrhea developed in an individual calf, a fecal swab was taken for classification of the pathogens involved. An autopsy was performed on calves that died. Postmortem findings were recorded, and the cause of death was assessed. For every calf, the study ended with a final examination that was identical to the control examinations except for an additional weighing.

\section{Data Management and Quality Assurance}

The clinical trials were conducted in accordance with the Rules Governing Medical Products in the European Union Volume VII, the Guidelines for Testing of Veterinary Medical Products, and the Council Regulation No. 595/98 (guideline on Good Clinical Practice).

All data were collected by a veterinarian in accordance with the investigational protocol and recorded on numbered case report forms. A clinical research associate regularly checked the accuracy and completeness of the case report forms.

\section{Statistical Analysis}

The IFNS GmbH (Institute for Numerical Statistics, Cologne, Germany) conducted the biometric planning and statistical analysis. The computer program used for the statistical analysis was SPSS 8 (SPSS Inc., Chicago, IL). For the hypothesis-generating trial, a total of 80 calves per treatment group were required. For efficacy and tolerance analyses, all case report forms were assessed. All data were analyzed with descriptive statistical methods.

Taking into account the results of the hypothesisgenerating trial, a superiority of 30 percentage points for the treatment with the $E$. coli strain Nissle 1917 in comparison to placebo was set for the confirmatory trial. With a type I error of $\alpha=0.05$ and a type II error of $\beta=$ 0.1 , a required sample size of 63 animals per treatment group was calculated. The target criteria were compared using the 2 -sided Fisher's exact test and descriptive statistical methods. The data of the 2 studies were evaluated in intention-to-treat (all animals) and perprotocol (animals without serious protocol violation) analyses.

\section{RESULTS}

A total of 335 newborn calves were included in the 2 studies (Table 1). Of these, 172 were included in the hypothesis-generating trial (study I), and 163 were included in the confirmatory trial (study II). The entire population for both trials included 206 female and 129 male newborn calves. Because of protocol violations (e.g., incomplete case report forms, violation of inclusion or exclusion criteria), 25 calves had to be excluded from the per-protocol analyses (study I: $\mathrm{n}=14$; study II: $n=11$ ).

The primary target criterion for both trials was the number of calves developing diarrhea. The results are shown in Table 2. 
Table 1. Number of calves included in the intention-to-treat and perprotocol analyses in the 2 studies.

\begin{tabular}{llll}
\hline Study & Total & Placebo & $\begin{array}{l}\text { Escherichia } \\
\text { coli }\end{array}$ \\
\cline { 3 - 4 } & & & (no.) \\
\cline { 3 - 4 } I & & 89 & 83 \\
$\quad$ Intention-to-treat analysis & 172 & 89 & 79 \\
$\quad$ Per-protocol analysis & 158 & 79 & 82 \\
II & & & 76 \\
$\quad$ Intention-to-treat analysis & 163 & 81 & 76 \\
$\quad$ Per-protocol analysis & 152 & 76 \\
\hline
\end{tabular}

In both studies, the rate of occurrence of diarrhea was considerably lower under treatment with $E$. coli strain Nissle 1917. In the hypothesis-generating study, 58 calves $(65.2 \%)$ of the intention-to-treat population developed diarrhea under placebo but only $22(26.5 \%)$ under $E$. coli strain Nissle 1917. This difference of 38.7 percentage points corresponds to a reduction rate of $>50 \%$. Within the per-protocol population, the corresponding figures were 37.4 percentage points and a more than $50 \%$ reduction rate.

In the second study, the therapeutic efficacy of $E$. coli strain Nissle 1917 was statistically substantiated. Diarrhea occurred within the intention-to-treat population in 51 cases $(63.0 \%)$ under placebo and in 10 cases (12.2\%) under E. coli strain Nissle 1917. Within the per-protocol population, the corresponding figures were 48 calves $(63.2 \%)$ under placebo and 10 calves $(13.2 \%)$ under $E$. coli strain Nissle 1917. This difference was statistically significant $(P<0.001)$.

Severity and duration of diarrhea were assessed and documented via the consistency of feces during the first 20 d of life. Under treatment with $E$. coli strain Nissle 1917, the number of days with normal feces was increased, and the number of days with changed fecal consistency was decreased. These results are shown in Table 3. In the placebo group, the average number of days with each consistency per calf were as follows: 1 ) 10.3 normal; 2) 6.7 soft but retaining its form; 3) 2.4 loose, not retaining its form; and 4) 0.6 watery. The

Table 2. Results for the primary target variable of number of calves developing diarrhea.

\begin{tabular}{lllll}
\hline Study & \multicolumn{2}{c}{ Placebo } & \multicolumn{2}{c}{$\begin{array}{c}\text { Escherichia } \\
\text { coli }\end{array}$} \\
\hline & $($ no. $)$ & $(\%)$ & $($ no. $)$ & $(\%)$ \\
I & & & & \\
$\quad$ Intention-to-treat analysis & 58 & 65.2 & 22 & 26.5 \\
$\quad$ Per-protocol analysis & 49 & 62.0 & 21 & 26.6 \\
II & & & & \\
$\quad$ Intention-to-treat analysis & 51 & 63.0 & 10 & $12.2 *$ \\
Per-protocol analysis & 48 & 63.2 & 10 & $13.2 *$ \\
\hline
\end{tabular}

*Escherichia coli vs. placebo significant at $P<0.001$ level.
Table 3. Results for the assessment of diarrhea.

\begin{tabular}{lcc}
\hline Feces consistency & Placebo & $\begin{array}{l}\text { Escherichia } \\
\text { coli }\end{array}$ \\
\hline & & (avg. d per calf) \\
Normal & 10.3 & 13.1 \\
Soft, but retaining its form & 6.7 & 5.8 \\
Loose, not retaining its form & 2.4 & 1.0 \\
Watery & 0.6 & 0.2 \\
\hline
\end{tabular}

corresponding figures for the E. coli strain Nissle 1917 group were 13.1, 5.8, 1.0, and 0.2 , respectively.

For the microbiological classification of pathogens that might have caused the diarrhea, a total of 125 fecal swabs were taken from animals with diarrhea. In 72 swabs, only clinically unspecific results were found: 20 swabs with Candida glabrata, 5 with Salmonella serovars (one identified as var. Copenhagen), 16 with Cryptosporidia spp., and 79 with Rotavirus. Multiple identifications were possible. However, a specific pathogen could not be identified as the cause of the cases of diarrhea observed in the studies.

Table 4 shows the results of the analyses of the secondary target criteria in both trials: cases of death, illnesses except diarrhea, and concomitant medication.

During the observation period, cases of death were observed in both treatment groups in the 2 trials; under placebo 18 calves (10.6\%) and under $E$. coli strain Nissle 191712 calves $(7.3 \%)$.

The following main causes of death were documented: circulatory (placebo: $\mathrm{n}=2 ; E$. coli strain Nissle 1917: $\mathrm{n}=0$ ), intestinal (placebo: $\mathrm{n}=9 ; E$. coli strain Nissle 1917: $\mathrm{n}=3$ ), respiratory disorders (placebo: $\mathrm{n}=1 ; E$. coli strain Nissle 1917: $\mathrm{n}=2$ ), mycotoxin intoxication (placebo: n $=0 ; E$. coli strain Nissle 1917: n $=5$ ), euthanasia because of congenital disorders or accidents (placebo: $\mathrm{n}=2 ; E$. coli strain Nissle 1917: $\mathrm{n}=1$ ), and unclear circumstances (placebo: n $=4 ; E$. coli strain Nissle 1917: $\mathrm{n}=1$ ). Multiple causes were possible. In addition, 2 calves in the placebo group in study II died immediately

Table 4. Results for the secondary target criteria.

\begin{tabular}{lcccc}
\hline $\begin{array}{l}\text { Secondary target } \\
\text { criteria }\end{array}$ & Placebo & \multicolumn{3}{c}{$\begin{array}{c}\text { Escherichia } \\
\text { coli }\end{array}$} \\
\hline & (no.) & $(\%)$ & (no.) & $(\%)$ \\
& & & & \\
Cases of death & 18 & 10.6 & 12 & 7.3 \\
$\quad$ Intention-to-treat analysis & & & & \\
$\quad \begin{array}{l}\text { Calves with "illnesses except diarrhea" } \\
\quad \text { Intention-to-treat analysis }\end{array}$ & 90 & 52.9 & 68 & 41.2 \\
$\quad$ Per-protocol analysis & 71 & 45.8 & 51 & 32.9 \\
$\quad \begin{array}{l}\text { Calves receiving concomitant medication } \\
\text { Intention-to-treat analysis }\end{array}$ & 121 & 71.2 & 95 & 57.6 \\
$\quad$ Per-protocol analysis & 108 & 69.7 & 88 & 56.8 \\
\hline
\end{tabular}


after completing the trial because of circulatory disorders or dehydration during bronchopneumonia.

Although 5 of 12 calves in the $E$. coli strain Nissle 1917 group died of mycotoxin intoxication (due to deoxynivalenol and zearalenon), the overall mortality rate was $33.3 \%$ lower in the population treated with $E$. coli strain Nissle 1917. Postmortem findings of calves on which an autopsy was conducted revealed no specific differences between the 2 treatment groups.

The following "illnesses except diarrhea" were documented: ataxia, dyspnea, bronchitis or bronchopneumonia, colic, keratoconjunctivitis, omphalitis, rhinitis, and tympania.

Within the intention-to-treat population, 90 animals (52.9\%) of the placebo group suffered from such illnesses, whereas in the group treated with the $E$. coli strain Nissle 1917 only 68 calves (41.2\%) suffered from such diseases. The corresponding per-protocol analysis revealed 71 cases $(45.8 \%)$ under placebo and 51 cases (32.9\%) under E. coli strain Nissle 1917. Depending on the population (i.e., intention-to-treat or per-protocol), the rate of "morbidity except diarrhea" was 24.4 to $28.2 \%$ lower for the animals treated with $E$. coli strain Nissle 1917.

Regarding the use of concomitant medication, a similar beneficial effect of the treatment with $E$. coli strain Nissle 1917 was documented. In the intention-to-treat analysis, 121 calves $(71.2 \%)$ in the placebo group and 95 calves $(57.6 \%)$ in the $E$. coli strain Nissle 1917 group received such medication. The corresponding figures for the per-protocol population were $108(96.7 \%)$ and 88 (56.8\%). Under treatment with $E$. coli strain Nissle 1917 , the rate of administered concomitant medication was 21.5 and $18.5 \%$ lower, respectively.

As assessed by the veterinarian, the calves treated with E. coli strain Nissle 1917 showed better general health status. In the study I placebo group, $4.0 \%$ of all observation days were classified as days with a "bad health condition." In the E. coli strain Nissle 1917 group, only $1.1 \%$ of the observation days were classified in this category. The corresponding figures for study II were $2.8 \%$ under placebo and $0.9 \%$ under $E$. coli strain Nissle 1917.

In both trials, the study medication was well tolerated, and no statistically relevant tolerance-related differences between the 2 treatment groups were found.

\section{DISCUSSION}

Calf diarrhea is the predominant disease during the first 30 to $50 \mathrm{~d}$ of life. Rearing, feeding, and health management on the farms influence the risk of disease, but they do not provide full protection (Frank and Kaneene, 1993; Wright et al., 1995). The main infectious causes of neonatal calf diarrhea are endemic in most calf-rearing establishments or brought in from outside. The crowding of animals originating from different locations is one of the most common factors leading to the spreading of pathogens resulting in changes of the intestinal microflora and, subsequently, the appearance of diseases.

Fluid and electrolyte replacement therapies are useful for solving the 2 acute problems, dehydration and electrolyte loss, but they are only symptomatic treatments. Antibiotics and antimicrobial drugs can be used specifically as causal therapy, but treatment must often be initiated without the cause of the diarrhea being known. The consequences of such unspecific treatments are reduction of the susceptibility of bacterial strains, disorders of the gastrointestinal microecosystem, and enrichment of resistant or unsusceptible organisms such as protozoa or yeasts (Elad et al., 1998). These effects are leading to an increasingly serious resistance problem. Because of this, it is important to develop new therapeutic alternatives.

One alternative to prevent and treat infections of the intestinal tract without causing resistance problems is the use of probiotics. In particular, the beneficial effects of bacilli (Kyriakis et al., 1999), bifidobacteria (Apgar et al., 1993; Abe et al., 1995), lactobacilli (Avila et al., 1995; Salminen et al., 1996), streptococci (Avila et al., 1995), and yeasts have been investigated intensively. Although oral administration of nonpathogenic $E$. coli strains had already been proposed by Nissle (1918) as a prophylactic measure against enteric infections, probiotic $E$. coli bacteria are only rarely mentioned in veterinary medicine. Zhao et al. (1998) described the effect of probiotic $E$. coli bacteria in reducing the carriage of enterohemorrhagic $E$. coli in cattle.

In general, probiotics promote establishment and stabilization of a beneficial gut flora and at the same time inhibit growth of pathogenic bacteria in the intestine. Mostly, they are commercially used as food additives. Observed positive effects are increased growth rates, improved food exploitation and conversion, improved milk performance and quality, and increased egg production. Most of these effects were observed in uncontrolled trials, however, and therefore have not been scientifically verified. This means that there is a lack of controlled clinical data. In addition, at the time these studies were conducted, no probiotic was classified as a medical drug, and none was available for the indication of prophylaxis and/or therapy of neonatal calf diarrhea.

In the studies presented here, the effect of the probiotic E. coli strain Nissle 1917 on prophylaxis and therapy of neonatal calf diarrhea under field conditions was investigated. Both studies were placebo-controlled and 
conducted in accordance with the Rules Governing Medical Products in the European Union for testing of veterinary medical products (guideline on Good Clinical Practice).

The analysis of the main target criterion showed that the administration of $E$. coli strain Nissle 1917 had a strong beneficial effect on the rate of animals fallen ill with diarrhea. Compared with the placebo group, a reduction of the diarrhea rate by $>50 \%$ (i.e., 38.7 percentage points lower incidence) was achieved in study I (intention-to-treat population) in the group treated with $E$. coli strain Nissle1917 (Table 2). This result is of clinical significance. In study II (intention-to-treat population), diarrhea occurred at a rate of $63.0 \%$ under placebo and $12.2 \%$ under $E$. coli strain Nissle 1917. This difference of 50.8 percentage points was statistically significant $(P<0.001)$.

Avila et al. (1995) were able to reduce the rate at which diarrhea occurred in newborn calves from 68.9 to $20.0 \%$ via combined vaccination and administration of Lactobacillus acidophilus. In our studies, a slightly greater reduction was achieved via the administration of $E$. coli strain Nissle 1917 alone.

In addition, diseased calves of the $E$. coli strain Nissle 1917 group showed a milder clinical course and a shorter period of sickness, resulting in fewer days per calf with changed fecal consistency and a reduction of concomitant medication. Animals treated with placebo showed a higher morbidity for "diseases except diarrhea" and received more concomitant medication such as cardiacs, antispasmodics, and antibiotics (Tables 3 and 4). These findings seem to suggest that administration of the probiotic strain not only prevents the development of diarrhea but also improves the general health status of the animals.

The number of deaths in the $E$. coli strain Nissle 1917 group compared with that in the placebo group also revealed the marked effect of the study medication. The evaluation of deaths was quantitative only and did not take the etiological aspects into account, which had a negative effect on the assessment of the efficacy of $E$. coli strain Nissle 1917. In particular, the quantitative data on mortality do not reveal that during the study II 5 of the calves in the $E$. coli strain Nissle 1917 group in one study center died after a peracute poisoning of mycotoxin due to an extreme infestation of the supplementary food with the fusarial toxins deoxynivalenol and zearalenon. Without these 5 cases, only 7 deaths would have occurred in the group treated with $E$. coli strain Nissle 1917. The resulting mortality rate would then have been only $4.2 \%$, resulting in a reduction of $61.1 \%$ in comparison to placebo.

Administration of viable cells of $E$. coli strain Nissle 1917 during the first days of life seems to influence the development of the intestinal flora in the direction of a healthy equilibrium. The mechanisms responsible for this beneficial effect are not yet understood. The known antagonistic (Lorenz and Schulze, 1996; Lodinová-Zádniková and Sonnenborn, 1997) and immunostimulating (Hockertz, 1997) effects of E. coli strain Nissle 1917 and its interference via occupation and masking of receptors of the intestinal mucosa are thought to prevent adhesion of microorganisms. This may protect against colonization of the intestine by potential pathogens and thus against the development of diarrhea.

The results of the studies presented in this article and additional studies led to the registration of $E$. coli strain Nissle 1917 as a drug for the prophylaxis of neonatal calf diarrhea in Germany. Since 2001, the strain has been marketed under the brand name Ponsocol and is commercially available to veterinarians and farmers.

\section{CONCLUSION}

In the 2 independent studies performed, the prophylactic use of E. coli strain Nissle 1917 in suckling calves led to a significant reduction in the frequency of diarrheal disease. Therapeutic administration to calves already ill with diarrhea resulted in a significant shortening of the duration of the disease. The clinical course of the disease was milder under medical treatment with $E$. coli strain Nissle 1917 than in the control animals receiving placebo. The necessity for additional dietary measures as well as for the application of additional pharmaceuticals-especially those exerting antimicrobial effects-was markedly reduced. Therefore, the risk of pharmaceutical derivatives in the animal body and the danger of the development of pharmaceutical-induced bacterial resistance can be reduced to a minimum. The study medication was well tolerated. No clinically relevant side effects were observed in either of the studies.

\section{REFERENCES}

Abe, F., N. Ishibashi, and S. Shimamura. 1995. Effect of administration of bifidobacteria and lactic acid bacteria to newborn calves and piglets. J. Dairy Sci. 78:2838-2846.

Apgar, G. A., E. T. Kornegay, M. D. Lindemann, and C. M. Wood. 1993. The effect of feeding various levels of Bifidobacterium globosum A on the performance, gastrointestinal measurements, and immunity of weanling pigs and on the performance and carcass measurements of growing-finishing pigs. J. Anim. Sci. 71:2173-2179.

Avila, F. A., A. C. Paulillo, R. P. Schocken-Iturrino, F. A. Lucas, A. Orgaz, and J. L. Quintana. 1995. A comparative study of the efficiency of a pro-biotic and the anti-K99 and anti-A14 vaccines in the control of diarrhea in calves in Brazil. Rev. Elev. Med. Vet. Pays Trop. 48:239-243.

Bendali, F., H. Bichet, F. Schelcher, and M. Sanaa. 1999. Pattern of diarrhoea in newborn beef calves in southwest France. Vet. Res. 30:61-74.

Blum, G., R. Marre, and J. Hacker. 1995. Properties of Escherichia coli strains of serotype 06. Infection 23:234-236. 
Blum-Oehler, G., S. Oswald, K. Eiteljorge, U. Sonnenborn, J. Schulze, W. Kruis, and J. Hacker. 2003. Development of strain-specific PCR reactions for the detection of the probiotic Escherichia coli strain Nissle 1917 in fecal samples. Res. Microbiol. 154:59-66.

Cornaglia, E. M., F. M. Fernandez, M. Gottschalk, M. E. Barrandeguy, A. Luchelli, M. I. Pasini, L. J. Saif, J. R. Parraud, A. Romat, and A. A. Schudel. 1992. Reduction in morbidity due to diarrhea in nursing beef calves by use of an inactivated oil-adjuvanted rotavirus-Escherichia coli vaccine in the dam. Vet. Microbiol. 30:191-202.

Elad, D., J. Brenner, A. Markovics, B. Yakobson, S. Shlomovitz, and J. Basan. 1998. Yeasts in the gastrointestinal tract of preweaned calves and possible involvement of Candida glabrata in neonatal calf diarrhea. Mycopathologia 141:7-14.

Frank, N. A., and J. B. Kaneene. 1993. Management risk factors associated with calf diarrhea in Michigan dairy herds. J. Dairy Sci. 76:1313-1323.

Fric, P. 2002. Probiotics in gastroenterology. Z. Gastroenterol. 40:197-201.

Gedek, B. 1986. Probiotika in der Tierernährung: Wirkungen auf Leistung und Tiergesundheit. Kraftfutter 3:80-84.

Grozdanov, L., U. Zähringer, G. Blum-Oehler, L. Brade, A. Henne, Y. A. Knirel, U. Schombel, J. Schulze, U. Sonnenborn, G. Gottschalk, J. Hacker, E. T. Rietschel, and U. Dobrindt. 2002. A single nucleotide exchange in the wzy gene is responsible for the semirough O6 lipopolysaccharide phenotype and serum sensitivity of Escherichia coli strain Nissle 1917. J. Bacteriol. 184:5912-5925.

Hockertz, S. 1997. Augmentation of host defense against bacterial and fungal infections of mice pretreated with the non-pathogenic Escherichia coli strain Nissle 1917. Arzneim. Forsch./Drug Res. 47:793-796.

Kruis, W., E. Schútz, P. Fric, B. Fixa, G. Judmaier, and M. Stolte. 1997. Double-blind comparison of an oral Escherichia coli preparation and mesalazine in maintaining remission of ulcerative colitis. Aliment. Pharmacol. Therap. 11:853-858.

Kuzela, L., M. Kascak, and A. Vavrecka. 2001. Induction and maintenance of remission with nonpathogenic Escherichia coli in patients with pouchitis. Am. J. Gastroenterol. 96:3218-3219.

Kyriakis, S. C., V. K. Tsiloyiannis, J. Vlemmas, K. Sarris, A. C. Tsinas, C. Alexopoulos, and L. Jansegers. 1999. The effect of probiotic LSP 122 on the control of post-weaning diarrhoea syndrome of piglets. Res. Vet. Sci. 67:223-228.

Lodinová-Zádniková, R., and U. Sonnenborn. 1997. Effect of preventive administration of a nonpathogenic Escherichia coli strain on the colonization of the intestine with microbial pathogens in newborn infants. Biol. Neonate 71:224-232.

Lorenz, A., and J. Schulze. 1996. Establishment of E. coli Nissle 1917 and its interaction with Candida albicans in gnotobiotic rats. Mikroökologie und Therapie. 24:45-51.

Malchow, H. A. 1997. Crohn's disease and Escherichia coli: A new approach in therapy to maintain remission of colonic Crohn's disease? J. Clin. Gastroenterol. 25:653-658.

Moellenbrink, M., and E. Bruckschen. 1994. Behandlung der chronischen Obstipation mit physiologischen Escherichia-coli-Bakterien. Ergebnisse einer klinischen Studie zur Wirksamkeit und Verträglichkeit der mikrobiologischen Therapie mit dem E.-coliStamm Nissle 1917 (Mutaflor). Med. Klin. 89:587-593.

Nissle, A. 1918. Die antagonistische Behandlung chronischer Darmstörungen mit Colibakterien. Med. Klin. 2:29-33.

Reid, G., and R. Friendship. 2002. Alternatives to antibiotic use: Probiotics for the gut. Anim. Biotechnol. 13:97-112.

Rembacken, B. J., A. M. Snelling, P. M. Hawkey, D. M. Chalmers, and A. T. R. Axon. 1999. Non-pathogenic Escherichia coli versus mesalazine for the treatment of ulcerative colitis: A randomised trial. Lancet 354:635-639.

Reuter, G. 1997. Present and future of probiotics in Germany and in central Europe. Bioscience Microflora 16:43-51.

Salminen, S., E. Isolauri, and E. Salminen. 1996. Clinical uses of probiotics for stabilizing the gut mucosal barrier: Successfu. strains and future challenges. Antonie Van Leeuwenhoek 70:347-358

Sonnenborn, U., and R. Greinwald. 1991 Pages 55-68 in Beziehungen zwischen Wirtsorganismus und Darmflora unter besonderer Berücksichtigung von Physiologie und Funktion der normalen Escherichia-coli-Flora. Schattauer Verlag GmbH, Stuttgart, Germany.

Stentebjerg-Olesen, B., T. Chakraborty, and P. Klemm. 1999. Type 1 fimbriation and phase switching in a natural Escherichia coli fimB null strain, Nissle 1917. J. Bacteriol. 181:7470-7478.

Virtala, A. M., G. D. Mechor, Y. T. Gröhn, and H. N. Erb. 1996. Morbidity from nonrespiratory diseases and mortality in dairy heifers during the first three months of life. JAVMA 208:20432046 .

Wright, A. K., R. Giger, T. M. Arnold, and E. D. Janzen. 1995. An episode of diarrhea in calves of a well-managed dairy herd. Can. Vet. J. 36:36-38.

Zhao, T., M. P. Doyle, B. G. Harmon, C. A. Brown, P. O. Mueller, and A. H. Parks. 1998. Reduction of carriage of enterohemorrhagic Escherichia coli 0157:H7 in cattle by inoculation with probiotic bacteria. J. Clin. Microbiol. 36:641-647. 